\title{
Conceptual Approach to the Use of Information Acquired in Social Media for Medial Decisions
}

\author{
Masuma Mammadova ${ }^{1 *}$ \\ (iD) 0000-0002-2205-1023
}

\section{Zarifa Jabrayilova ${ }^{1}$}

(D) 0000-0002-9661-5805

\author{
Aytac Isayeva ${ }^{1}$ \\ (iD) 0000-0002-9573-4845 \\ ${ }^{1}$ Institute of Information Technology of the National Academy of Sciences of Azerbaijan, Baku, AZERBAIJAN \\ *Corresponding author: mmg51@mail.ru
}

Citation: Mammadova, M., Jabrayilova, Z., \& Isayeva, A. (2020). Conceptual Approach to the Use of Information Acquired in Social Media for Medial Decisions. Online Journal of Communication and Media Technologies, 10(2), e202007. https://doi.org/10.29333/ojcmt/7877

\section{ARTICLE INFO}

Received: 18 Nov 2019

Accepted: 19 Feb 2020

\section{ABSTRACT}

A conceptual approach to the use of information collected in medical social media for decisionmaking is proposed. The formation of e-medicine has turned the medical social media environment into an important source of information for improving the medical decisionmaking process, taking into account public opinion. Referring to this source, the information collected to obtain the data essential for medical decision-making is classified, and the medical social media environment is segmented for user relations. The information collected in the physician-patient segment is taken as a research object, and the inquiries of e-patients in a number of national medical resources are statistically analyzed. Referring to the results and demographic data of e-patients, the activity of the stakeholders in medical social media is assessed, and the informative indicators for medical decisions are defined. The process of medical decision-making is formally described. The results of the study represent an innovative approach to the use of the results of statistical analysis of information collected in the national medical social media to improve medical decision-making. This approach constitutes the conceptual framework for a decision support system to improve the quality of health care, taking into account public opinion.

Keywords: medical social media, stakeholders, e-patient requests, informative indicators, medical decision-making

\section{INTRODUCTION}

At present, information technology and the Internet have created a basis for e-medicine which has penetrated all areas, including healthcare. The introduction of network technology and cloud computing technology, the creation of IoT-based health care systems have become an e-medical reality (Usak et al., 2019). The main indicators of the formation of e-medicine may include the modification of traditional communication sources and tools used by the physicians and patients to establish communication. To access the information the physicians and patients shift into the virtual environment (Islam et al., 2018). An obvious example of such a tool is medical social media. At present, there are many social media tools (Efimenko \& Horoshevskij, 2016): network platforms, blogs, micro blogs, wikis, data exchange sites, virtual reality, etc. These tools serve to improve professional networks, to develop public health programs, and to educate patients. Using social media resources, e-patients are supported by the experts and recovered patients. They post complaints and symptoms in a social network, and e-patients get the opportunity to check the validity of the diagnosis and treatment methods assigned to them (Mammadova \& Jabrayilova, 2019). Currently, the use

Copyright (c) $\mathbf{2 0 2 0}$ by authors; licensee OJCMT. This article is an open access article distributed under the terms and conditions of the Creative Commons Attribution License (http://creativecommons.org/licenses/by/4.0/). 
of crowdsourcing technologies support the patients to find analogical precedents (Chang \& Choi, 2016; Gallant et al., 2011; Swan, 2012). Therefore, e-patient gets an opportunity to participate in the decision-making process concerning the treatments. Today, the concept of "responsible patient", who is able to make independent decisions, is getting popular in the world. Involvement of a citizen in the protection of his/her own health has become an integral part of the policy of a number of states (Martino et al., 2017). Thus, the combination of patient-oriented medicine, social media and the Internet has opened a new era in medicine of the 21 st century.

At present, doctors using social media are able to: watch conferences and consiliums without leaving the workplace; hold discussions with colleagues; provide decision support in clinical situations for nurses; and involve professional staff to raise personal potential (Alguliyev, Aliguliyev \& Yusifov, 2018; Bridewell \& Das, 2011). Social media supports the physicians to make "wise decisions" based on the information generated by the crowd. Medical social media creates such opportunities for doctors and patients, and collected requests, medical inquiries become very significant information resource. They are used to increase the quality of medical services and the process of decision-making based on the statistical and content analysis of requests (Cesare, Grant \& Hawkins, 2017; Mammadova \& Isayeva, 2018). Use of analytics, including data mining, text mining, and big data analytics are also beneficial. They are assisting healthcare professionals in disease prediction, diagnosis, and treatment, resulting in an improvement in service quality and reduction in cost (Tomar \& Agarwal, 2013). Improving the process of medical decision-making using this source is of great significance and relevance in the context of e-medicine. Therefore, this article provides a conceptual description of obtaining the necessary information for medical decision-making by classifying and analyzing diverse, important and chaotic information collected in the medical social media.

\section{ANALYSIS OF LITERATURE}

Recent articles have been focusing on the rise of social media activity and even the emergence of their dependence on social media (Aksoy, 2018; Bronze-Aksan \& Akbay 2019; Simsek, Elciyar \& Kizilhan, 2019). On the other hand, an increase in social media activity in the community will make the data collected in this environment a valuable source of information for addressing various issues. Amit, Tejashree, and Swati (2014), Bollen, Mao, and Zeng (2011), Dalal and Zaveri (2014) emphasize that, based on the content analysis of the user requests, the potential customers, who need a particular product or service, can be identified. The demand for a particular product or service can be predicted, the citizens' attitude to a particular event, such as the election, can be explored. Haque and Rahman (2014), Nadali, Murad, and Kadir (2010), Vo and Ock (2012) use the fuzzy apparatus to automatically assess the users' requests in social media, given that the users' emotions are expressed through the words, i.e. in terms of quality rather than the quantity (Zadeh, 1975). The approaches proposed in these sources do not take into account the interaction of users with one another, and how many users generate the total requests. On the other hand, currently, one "authoritative" user in social networks can influence the formation of opinions of others, however, this point is not addressed in the reviewed sources. Luneva, Efremov, and Banokin (2015) proposes an approach to the use of fuzzy apparatus for automatic evaluation of user emotions. It takes into account the interactions of users and the indicator of the "impact" of "authoritative" users on others.

Medical facilities and physicians share the information related to new drugs, diseases, symptoms, treatment methods, etc. on social networks. This information makes millions of users connect the social media, making it a flexible platform for the study of user feedback. Krithika and Rosiline (2017) presents a two-phased structure for the analysis of the users' opinions related to the narcotic drugs used in the treatment of tumors. The comments of the forum participants are evaluated as negative and positive due to the frequency of the use of certain words and phrases. Based on the approach proposed in this source, an approach and automatic feedback assessment method referring to the user feedback on Twitter for the knowledge extraction is developed. Mengxue, Meizhuo, and Chen (2019) states that the fast detection of user feedback related to a certain drug may prevent large amounts of damage of the drug manufacturer. Social media is regarded as the most flexible source of information for acquisition of such information. The authors refer to the data collected in the physician-patient relations segment in an online social network environment to get feedback related to the drugs. To this end, the data collected on two Chinese social networks is used 
as key information. A method for feedback assessment based on the names of the drugs found in the texts and the content analysis is proposed.

Content analysis in the medical social media environment (Mammadova, Jabrayilova \& Isayeva, 2019; Tomar \& Agarwal, 2013) focus on the physician-patient relations. The study emphasizes the importance of these relations for improving the medical decision-making process and perfecting the health care system. Using the content analysis in the source the patients' opinions about the physicians and medical facilities are explored, and a three-level classification scheme is used to report the analysis results.

Generally, the studies devoted to the analysis of the data collected on social networks, including in medical social networks are often based on the content analysis of requests. Using content-based knowledge technologies they solve the problems associated with the data collected in medical social networks related to this or another area of medicine. Submitted article addresses the issue of necessary indicators acquisition for medical decision-making with the use of statistical analysis of the data collected in social media. It presents the statistical analysis of the requests of e-patients collected in the physician-patient relations segment of social media. The opportunities of accessing a number of informative indicators referring to demographic data submitted by the patients when registering in national medical networks are shown.

The goal of the article is to develop a conceptual framework for medical decision support in-country through the use of information collected in the medical social media environment. To achieve this goal, the following issues are to be addressed:

- classifying the data collected in medical social media environment;

- statistically analyzing the e-patient requests in the physician-patient relations segment;

- developing the informative indicators necessary for medical decision making;

- developing a formal description of medical decision making.

\section{CLASSIFICATION OF THE DATA COLLECTED IN MEDICAL SOCIAL MEDIA ENVIRONMENT}

Campanini (2016) states that, currently, $26 \%$ of global clinics are operating on social networks, $46 \%$ of which use Youtube, $84 \%$ - Facebook, 64\% - Twitter, and 12\% - blogs. A survey conducted in 2017 revealed that $80 \%$ of world physicians used social media for their personal contacts, professional communications and research (Bhaskar, 2017; Campbell et al., 2016). A mass use of social networks has led to the emergence of professional medical societies on the Internet. One of them is Sermo, a social networking community for doctors (Fogelson, Rubin \& Ault, 2017). Developed in 2011, Doximity is a social networking community for doctors established. According to the 2015 report, this network has more than 500,000 members.

Medical social networks such as Doc2Doc, Ozmosis, Healtheva are considered to be the most sophisticated platforms for the communication between doctors and patients (Keckley, 2010; Khokhar, 2017). Networks, such as The Medical Directors Forum, QuantiaMD, Doctors Hangaut, Doc2Doc, are important platforms for healthcare providers to communicate and collaborate with each other and patients. Patients can benefit from the physicians' services by freely registering at the networks Medihost.ru, adam.com, qapsula.com, DoctorSpring, likar.info, doslovno.com, health.mail.ru. E-patients registered in these networks are required to provide information about their name, age, e-mail address, while qapsula.com and doslovno.com require them to provide information about their location.

Most patients use the following social networks and social media platforms: Smart Patients, Stupidcancering, e-patients.net, Woman Heart Support Community, babycenter, Daily Strength and Facebook, Twitter. Patientslikeme.com and treato.com are is supporting only e-patient societies. Patientslikeme.com is designed to analyze the types of therapy (including preparations), symptoms, possible complications, and other aspects based on the treatment experience of various diseases. One of the main issues of this resource is finding similar diagnosed patients and creating opportunities for users to examine their treatment experiences.

Study of various types of medical social media resources allows identifying their trends and main scopes (users). This, in turn, has resulted in the segmentation of users by their scopes and the development of certain 


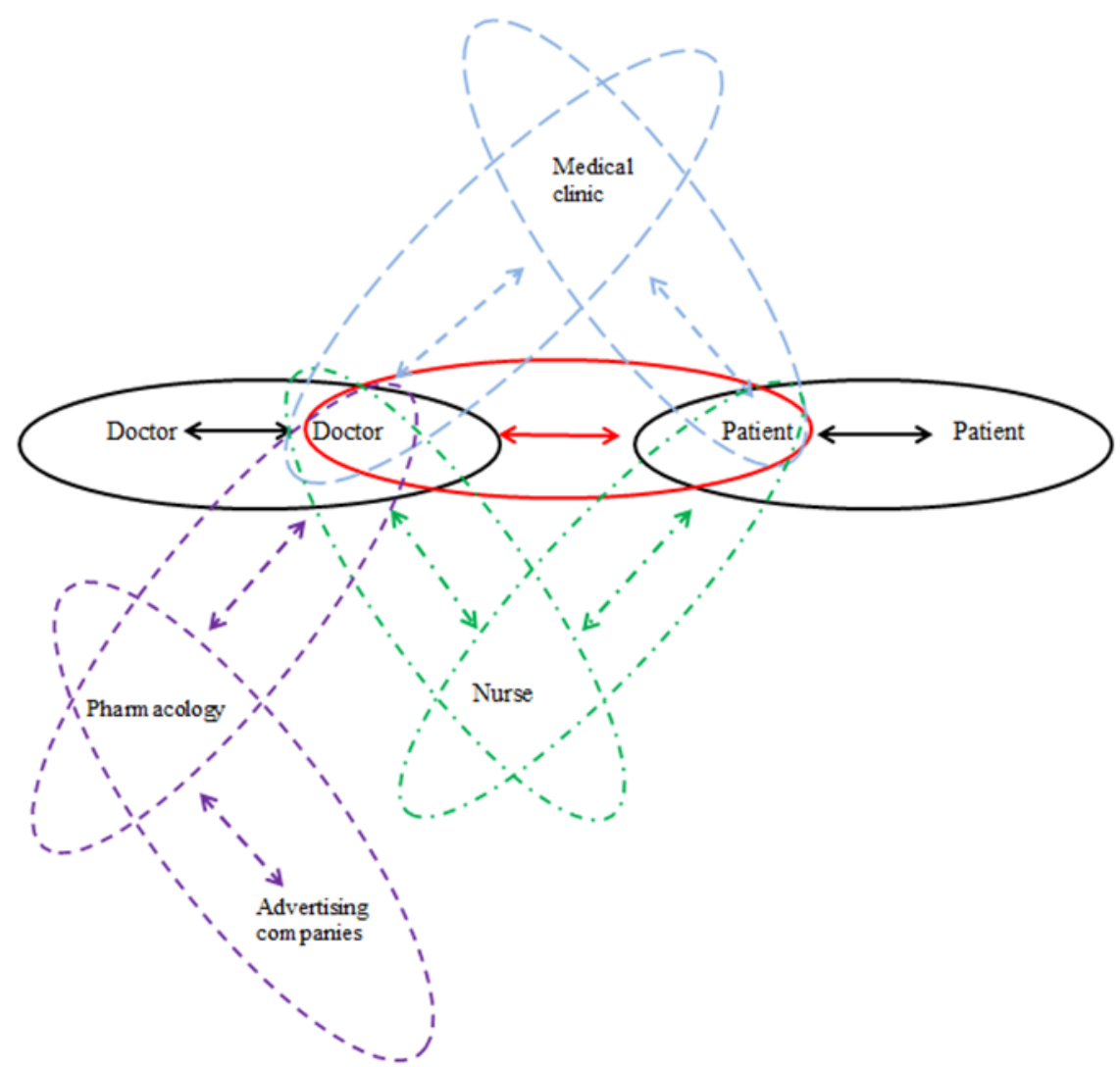

Figure 1. Relationships in medical social media

types of requests and relationships in the virtual space. Depending on these relationships and requests type, scenarios for decision-making can be set in the social media environment. As a result of the research, we have found the following relationships in medical social media (Mammadova \& Jabrayilova, 2019) (Figure 1):

- Physician-physician relations. Using social media, physicians can discuss effective treatment methods of various diseases with their colleagues. They can also provide professional advice on clinical situations, conduct scientific negotiations, develop their medical knowledge, and make accurate decisions in clinical situations. Simultaneously, physicians can watch real-time scientific workshops of more experienced professionals, their surgical operations and instructions.

- Physician-patient relations. Physicians use social media to communicate with patients, observe their health, give advises and monitor them.

- Patient-patient relations. Patients use social networks, mostly in search of necessary information. This information may include diseases, symptoms, diagnosis and treatment; medicines, their use, and adverse reactions; opinions of other patients about doctors and their treatment methods.

- Physician-pharmaceutical company relations. Physicians get information about the medications produced by pharmaceutical companies through social media and get acquainted with their instructions and adverse reactions. Accordingly, doctors prescribe new therapeutic treatments, share information on social media about the poor quality of certain medicines, and call pharmacists to stop producing these drugs.

- Pharmacist-advertising company relations. Social media provide successful business opportunities for pharmacology. Thus, pharmacists contract advertising companies, promote and sell medications in social media.

- Physician-patient-nurse relations. The therapeutic process depends largely on the relationship between physicians and nurses. The absence of mutual understanding and harmony in these relationships diminishes the quality of medical services. The emergence of social media has also brought innovations to the relations. Thus, online social technologies such as e-mail and computerized 
order have enabled physicians and nurses to communicate without interruptions. Accordingly, they can monitor the patient's treatment, and get information and follow doctors' instructions. At the same time, relationship between the patient and the nurse is also important.

- Physician-medical clinics relations. Social media is the most comfortable way to search and select medical staff for medical institutions. Managers of medical institutions get information about doctors visiting their pages on popular social networks (e.g. Facebook) or personal medical sites to identify the extent of their professionalism based on opinions of patients about their treatment methods, treatment results and experiences.

- Patient-medical clinics relations. Social media sites, patients can access the clinic, its address, contacts and detailed information about medical staff and appointment rules, including the web site of that clinic.

Depending on possible requests, feedbacks, the decision-making scenarios on improving the quality of medical care are developed taking into account the opinion of the mass. For example, based on the survey analytics, we can define which physician is most often referred to. Or what disease and medication are most often challenging, and so forth.

The two stakeholders interested in seeking information and receiving moral support in physician-patient relations segment include the patients and medical specialists. In this segment, the patients (or their guardians) may apply to doctors related to the problems with disease, health, any symptoms, medications, diagnosis and treatment methods, and so on. By analyzing the social media activity of physicians and epatients, as the most frequently addressed physician can be identified. Moreover, the most frequently referred field of medicine, the activity of female and male e-patients can also be identified for their age groups, gender, and territory.

A patient, in the patient-patient relations segment with a certain problem may send requests to find "selfsimilars", appointed diagnosis, treatment method, the effects and side effects of certain medications. In this case, the patients will have access to social support for decision-making on treatment options, as well as promote the spread of more effective, proven treatment methods, and so on.

Obviously, the analysis of all requests across all segments allows for the achievement of a set of indicators that are important for medical decisions. Thus, the surveys of the physician-patient relations segment are analyzed in the national media space. The aim is to obtain informative indicators for medical decision support based on the information collected from the medical social media environment.

\section{ANALYSIS OF PHYSICIAN-PATIENT RELATIONS SEGMENT IN MEDICAL SOCIAL MEDIA ENVIRONMENT}

Physician-patient relations segment in the medical social media environment of Azerbaijan is analyzed. Professional societies merging the doctors specialized in various medical fields on one portal are available in Azerbaijan. These social societies are "Həkim.tap", "həkimsən.az", "sağlamolun.az" and "doctormap.az". Developed in 2012, Doctormap social society clearly indicates the address of physicians and clinics and regularly publishes scientific articles and clinical observations on web pages. The video section of the social network "Həkimtap.az" uploads videoconferences, lectures and training videos and interviews with famous physicians. Detailed information about the doctors is available to the patients through the "doctor-search" section of these networks. The patients can also ask those physicians questions. Other social societies gathering together specialized doctors in the same field of medicine, for example "DentStar.az", are also available.

Thus, based on the information collected, the segment of physician-patient relations is analyzed to determine the activity of the country, physicians and patients in this environment. This research refers to one of the medical social media societies, namely tibb.az as a database. Note that it has a broad user audience with 1966278 members in Azerbaijan. Statistical and content analysis is conducted on ophthalmology, gastroenterology and hepatology, and the requests on these areas are analyzed (Figure 2). 


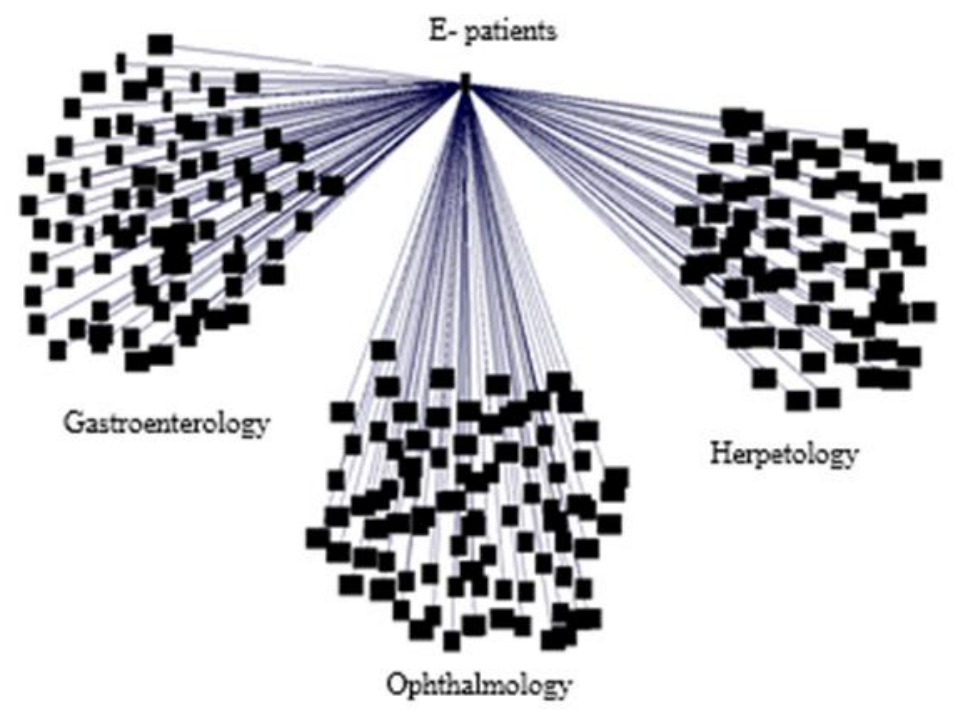

Figure 2. Description of e-patients' requests to ophthalmology, gastroenterology and hepatology at tibb.az portal

Table 1. Clustering the requests to tibb.az portal on ophthalmology, gastroenterology and hepatology

\begin{tabular}{|c|c|c|c|}
\hline Stakeholders & & & Requests to Medical \\
\hline Medical fields & Personally & Guardians & Specialists \\
\hline Ophthalmology & 200 & 138 & 338 \\
\hline Gastroenterology & 588 & 83 & 671 \\
\hline Hepatology & 238 & 60 & 298 \\
\hline
\end{tabular}

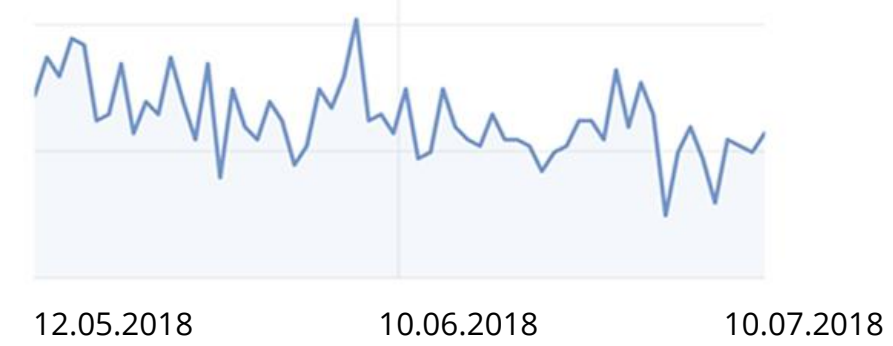

Figure 3. Dynamics of patients' requests to the Facebook medical group "Online medical consultation" for the period of 12.05.2018-10.07.2018

The statistics on abovementioned three medical fields for both years (from June 2016 to September 2018) collected and available in the physician-patient segment of tibb.az portal is assigned (Table 1).

One of the most commonly referred sources of medical information in Azerbaijan is Facebook. In this study, it is referred to the requests recorded at online medical consultation group "Online medical consultation". This group has 61373 members. The aim is to define the number of requests at Facebook and to identify their distribution by medical fields. The analysis of the activities of the patients in this group for 2 months (12.05.2018-10.07.2018) shows that 1512 requests are registered (Figure 3 ).

Out of them, 1400 are related to diseases and addressed to the doctors on 14 medical specialties. Distribution of the requests on 14 medical specialties is given in Figure 4. 


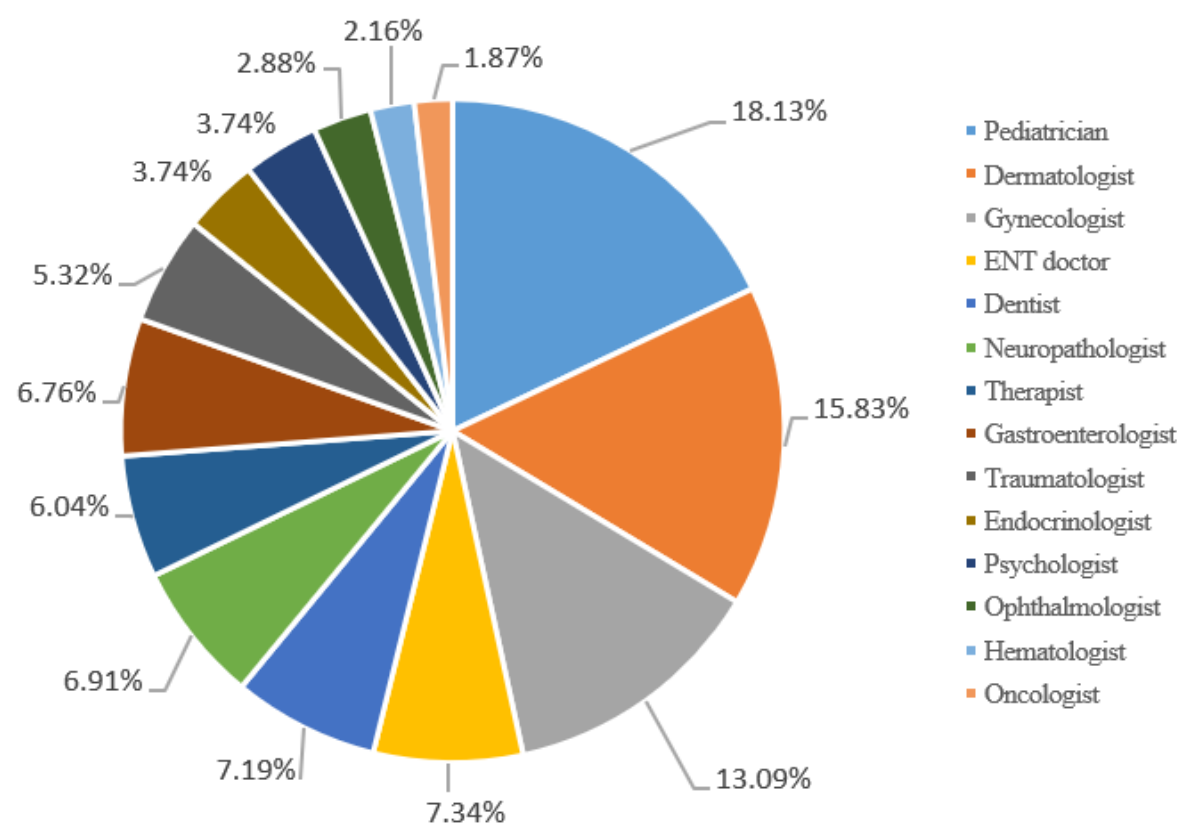

Figure 4. Distribution of the requests the Facebook medical group "Online medical consultation" on 14 medical specialties

According to the diagram, the patients' requests are mostly on pediatrics (18.13\%), dermatology (15.83\%) and gynecology (13.09\%). The study reveals that majority of applicants are female.

Thus, the findings from the research enable to produce a formal description of the evaluation of the activity in the medical social media environment in Azerbaijan.

\section{INITIAL INDICATORS FOR DECISION MAKING AVAILABLE AT THE PHYSICIAN- PATIENT SEGMENT OF THE SOCIAL MEDIA ENVIRONMENT}

As mentioned above, the requests to the medical social media environment are formulated directly by the patients themselves and their guardians. Assume that $M$ is the number of overall requests. Obviously, the overall requests are based on the user requests on different medical fields (e.g. ophthalmology, gastroenterology, hepatology, etc.), i.e.: $M=\sum_{i=1}^{n} M_{i}$.

Here, $M_{i}$ is the number of requests on the medical field $i$, and $n$ is the number of medical fields. On the other hand, many portals (e.g., tibb.az, həkim.az, Facebook.com, etc.) are now available in the medical social media environment in each country, and the number of requests $M$ is based on the patients requests on different fields of medicine, as follows:

$$
M=\sum_{t=1}^{T} M_{i}=\sum_{t=1}^{T} \sum_{i=1}^{n} M_{t i}
$$

Here, $T$ is the number of portals in the medical social media environment, $M_{t i}$ is the number of applications on the medical field $i$ at portal $t$.

Assume that $D=\left\{D_{i}, i=\overline{1, n}\right\}$ is a set of medical specialists working in the medical social media environment (physicians, in this case), and $D_{i}$ is a set of physicians working on the medical field $i$ in the medical social media environment, consequently: $D_{i}=\left\{D_{i j}, j=\overline{1, m}\right\}$.

Where $m$ is the number of doctors working in the medical field $i$ in the social media environment.

Then, $n \times m$ will be the total number of doctors working in the social media environment.

Admit that $M_{D_{i}}$ is the number of requests to doctors in the medical field $i$, and $M_{D_{i j}}$ is the number of requests to the doctor $D_{i j}$ in the medical field $i$. 
Thus, the followings are offered to assess the activity at the medical social media environment based on the informative indicators available at the physician-patient relationship segment of the medical social portals.

Based on these informative indicators, the followings are suggested to assess the activity in the medical social media environment and to define the initial data required for medical decision-making.

\section{Indicators for the Evaluation of Activity in Medical Social Media Environment}

1. Activity of physicians in the medical social media in country. Knowing the number of doctors $D_{n \times m}$ in the medical social media space and the number of doctors $D_{\text {st }}$ in country (for exp. in Azerbaijan source (Cities and districts of the Republic of Azerbaijan. Key indicators of healthcare in cities and districts of the country at the beginning of 2018. www.stat.gov.az/source/healthcare/az/001_5_2.x/s)), activity of doctors in the medical social media in Azerbaijan can be assessed based on the following formula:

$$
F_{D}=D_{n \times m} / D_{s t}
$$

2. Activity of physicians in the social media by medical fields in country. Activity of physicians in social media can be defined as follows based on the number of doctors ( $m$ if $D_{i}=\left\{D_{i j}, j=\overline{1, m}\right\}$ ) in social media in the medical field $i$ and the number of doctors (source www.stat.gov.az/source/healthcare/az/001_5_2.x/s) in this medical field:

$$
F_{D_{i}}=m / D_{i s t}, i=\overline{1, n}
$$

3. Social media activity in the field of medicine in country. If $M$ is the number of total requests and $M_{i}$ is the number of requests in medical fields, then the social media activity in the medical fields can be evaluated based on the following formula:

$$
F_{i}=M_{i} / M, i=\overline{1, n}
$$

Assigning $F_{i}^{*}=\max \left\{M_{i}, i=\overline{1, n}\right\}$, the medical spheres can be rated by social media activity.

4. Social media activity of physicians on medical fields. Based on the number of requests $M_{D i j}$ to each physician working in the medical field $i$ in social media and the number of overall requests $M_{i}$ in this medical field, the social media activity of physicians can be determined by the following formula:

$$
F_{D_{i j}}=M_{D_{i j}} / M_{i}
$$

Based on $F_{D_{i j}}^{*}=\left\{\max F_{D_{i j}}, j=\overline{1, m}\right\}$, the social media activity of physicians in the medical field $i$ can be rated.

5. Activity of physicians by medical portals. If $M_{t D i j}$ is the number of requests from a particular medical portal to any physician, and $M_{D i j}$ is the number of overall requests to this physician, activity of physicians by medical portals can be evaluated based on the following formula:

$$
F_{t_{D_{i j}}}=M_{t_{D_{i j}}} / M_{D_{i j}}
$$

Based on $F_{t D_{i j}}^{*}=\left\{\max F_{t D_{i j}}, t=\overline{1, T}, i=\overline{1, n}, j=\overline{1, m}\right\}$, the most frequently requested physician by the portal $t$ can be defined, and the physicians can be rated by this portal.

\section{Evaluation of the Users' Activity in the Medical Social Media based on Demographic Indicators}

1. Users' activity in the medical social media with regard to their gender. As mentioned above, the patients (or their guardians) have to specify gender information when registering in the medical portal. This information allows to define the distribution of the overall requests by the users' gender. That is: $M=M_{q}+M_{k}$.

Where $M_{q}$ denotes the number of requests of female patients and $M_{k}$ denotes the number of requests of male patients in the medical social network.

Then: $F_{q}=M_{q} / M$ is the indicator of social media activity female patients, while $F_{k}=M_{k} / M$ denotes the respective indicator for male patients.

2. Social media activity by medical fields with regard to the patients' gender. The overall requests in the medical social media environment are shaped based on the requests of female and male patients to various fields of medicine. Given this, the number of overall requests can be denoted as follows: 


$$
M=M_{q}+M_{k}=\sum_{i=1}^{n} M_{i q}+\sum_{i=1}^{n} M_{i k}
$$

Then based on $F_{i q}=M_{i q} / M_{q}$, activity of female patients by the fields of medicine can be evaluated. The medical field most frequently requested by female patients can be defined based on $F_{i q}^{*}=\max \left\{F_{i q}, i=\overline{1, n}\right\}$, and according to this indicator, the rating of the medical fields can be determined.

Based on $F_{i k}=M_{i k} / M_{k}$, activity of male patients by the fields of medicine can be evaluated. The medical field most frequently requested by male patients can be defined based $\operatorname{on} F_{i k}^{*}=\max \left\{F_{i k}, i=\overline{1, n}\right\}$.

3. Medical social media activity with regard to the patients' age. The patients (or their guardians) using the medical portal specify age-related information when registering. This enable to classify the requests by the patients' age. In this regard, the patients are classified into the following age groups: Age group $A$ (under 24), age group $B$ (25-44) and age group $C$ (45 and above). Then, the overall requests will be same as the total number of requests of the patients of these age groups, i.e.: $M=M_{A}+M_{B}+M_{C}$.

Where:

$M_{A}$ denotes the number of patients from age group $A, M_{B}$ - from age group $B$, and $M_{C}$ - from age group $C$.

Then: $F_{A}=M_{A} / M$ indicates the activity of patients in the medical social media from age group $A, F_{B}=M_{B} / M$ from age group $B$, and $F_{C}=M_{C} / M$ - from age group $C$.

4. Activity of users in the medical social media with regard to their gender and age. The overall requests in medical social media are shaped based on the requests of female and male patients of different age groups. This can be expressed as follows:

$$
M=M_{A}+M_{B}+M_{C}=\left(M_{A q}+M_{A k}\right)+\left(M_{B q}+M_{B k}\right)+\left(M_{C q}+M_{C k}\right)
$$

Where:

$M_{A q}$ is the number of female patients from age group $A, M_{B q}$ - from age group $B$, number of $M c q$ - from age group $C$.

$M_{A k}$ is the number of male patients from age group $A, M_{B k}$ - from age group $B$, number of $M_{C k}$ - from age group C. On it:

$F_{A q}=M_{A q} / M_{A}$ indicates the social media activity of female patients from age group $A, F_{B q}=M_{q B} / M_{B}$ - from age group $B$, and $F_{C q}=M_{C q} / M_{C}$ - from age group $C$.

$F_{A k}=M_{A k} / M_{A}=F_{A}-F_{A q}$ indicates the social media activity of male patients from age group $A, F_{B k}=$ $M_{B k} / M_{B}=F_{B}-F_{B q}$-from age group $B$, and $F_{C k}=M_{C k} / M_{C}=F_{C}-F_{C k}$ - from age group.

5. Social media activity of patients by the medical fields according to their age. The overall requests to the medical social media environment in country are shaped based on the requests of the patients from different age groups on various medical fields, i.e.:

$$
M=M_{A}+M_{B}+M_{C}=\sum_{i=1}^{n} M_{i A}+\sum_{i=1}^{n} M_{i B}+\sum_{i=1}^{n} M_{i C}
$$

Then:

The social media activity of the patients by the fields of medicine from age group $A$ can be evaluated based on $F_{i A}=M_{i A} / M_{A}$, from age group $B$ - based on $F_{i B}=M_{i B} / M_{B}$, and from age group $C$ - based on $F_{i C}=M_{i C} / M_{C}$.

In this case, it is possible to determine the medical field most commonly referred by the patients from age group $A$ based on $F_{i A}^{*}=\max \left\{F_{i A}, i=\overline{1, n}\right\}$, from age group $B$ - based on $F_{i B}^{*}=\max \left\{F_{i B}, i=\overline{1, n}\right\}$, and from age group $C$ - based on $F_{i C}^{*}=\max \left\{F_{i C}, i=\overline{1, n}\right\}$, and according to this indicator, the medical fields can be rated.

6. Social media activity of patients by the medical fields with regard to their gender and age. Patients' requests from different age groups can be expressed in the following way:

$$
\begin{aligned}
& M_{i A}=M_{i A q}+M_{i A k} \\
& M_{i B}=M_{i B q}+M_{i B k} \\
& M_{i C}=M_{i C q}+M_{i C k}
\end{aligned}
$$

In this case: 
Activity of female patients by medical fields from age group $A$ can be evaluated based on $F_{i A q}=M_{i A q} / M_{i A}$, from age group $B$ - based on $F_{i B q}=M_{i B q} / M_{i B}$, from age group $C$ - based on $F_{i C q}=M_{i C q} / M_{i C}$.

The medical field most frequently requested by female patients from age group $A$ can be defined based on $F_{i A q}^{*}=\max \left\{F_{i A q}, i=\overline{1, n}\right\}$, from age group $B$ - based on $F_{i B q}^{*}=\max \left\{F_{i B q}, i=\overline{1, n}\right\}$, from age group $C$ - based on $F_{i C q}^{*}=\max \left\{F_{i C q}, i=\overline{1, n}\right\}$, and according to this indicator, the medical fields can be rated.

Activity of male patients by medical fields from age group $A$ can be evaluated based on $F_{i A k}=M_{i A k} / M_{i A}$, from age group $B$ - based on $F_{i B k}=M_{i B k} / M_{i B}$, from age group $C$ - based on $F_{i C k}=M_{i C k} / M_{i C}$.

The medical field most frequently requested by male patients from age group $A$ can be defined based on $F_{i A k}^{*}=\max \left\{F_{i A k}, i=\overline{1, n}\right\}$, from age group $B$ - based on $F_{i B k}^{*}=\max \left\{F_{i B k}, i=\overline{1, n}\right\}$, from age group $C$ - based on $F_{i C k}^{*}=\max \left\{F_{i C k}, i=\overline{1, n}\right\}$, and according to this indicator, the medical fields can be rated.

7. Medical social media activity of e-patients in the regions of country. As mentioned above, the patients (or their guardians) using a medical portal indicate the region (economic district, city) of the country when registering. This information allows to perform the distribution of requests to the medical social media across the regions, more precisely, the activity of e-patients by regions. If the number of regions in the country is $R$, then, the formulation of overall requests to in the medical social media environment by regions can be expressed as follows:

$$
M=M_{z_{1}}+\ldots+M_{z_{R}}=\sum_{r=1}^{R} M_{z_{r}}
$$

For example, according to key socio-economic indicators of economic regions in 2017 (www.stat.gov.az/source/regions/), the territory of Azerbaijan is divided into the 11 economic and regions and the formulation of overall requests to in the medical social media environment by regions can be expressed as follows: $M=M_{z_{1}}+\ldots+M_{z_{11}}=\sum_{r=1}^{11} M_{z_{r}}$.

In this case, $F_{z_{r}}=M_{z_{r}} / M, r=\overline{1, R}$.

The activity of medical social media can be determined based on the patients' regions.

Based on $F_{z r}^{*}=\max \left\{F_{z r}, r=\overline{1, R}\right\}$, we can determine the region from which e-patients most frequently refer to the medical social media environment, and get their rating.

8. Medical social media activity of users according to their gender and region of residence. If we refer to the requests to the medical social media environment by regions and with regard to the patients' gender, then the number of overall requests can be shown as follows:

$$
M=M_{z_{1}}+\ldots+M_{z_{R}}=\sum_{r=1}^{R} M_{z_{r}}=\sum_{r=1}^{R}\left(M_{q z_{r}}+M_{k z_{r}}\right)
$$

Here, $M_{q z_{r}}$ is the number of requests of female patients from the region $Z_{r}(r=\overline{1, R})$, whereas $M_{k z_{r}}$ is the number of requests of male patients.

Then, activity of female patients in the medical social network by the regions can be evaluated based on $F_{q z_{r}}=M_{q z_{r}} / M_{z_{r}}, r=\overline{1, R}$.

Based on $F_{q z_{r}}^{*}=\max \left\{F_{q Z_{r}}, r=\overline{1, R}\right\}$, we can determine the region which female patients most frequently refer from, and get the rating of the regions based on these indicators.

The medical social media activity of male patients by regions can also be evaluated analogically based on $F_{k z_{r}}=M_{k z_{r}} / M_{z_{r}}, r=\overline{1, R}$, and the region, which they most frequently refer from, can be defined based on $F_{k z_{r}}^{*}=\max \left\{F_{k z_{r}}, r=\overline{1, R}\right\}$.

9. The social media activity of patients according to the medical fields and by regions. The requests $b$ the medical fields are based on the requests from the regions. Given this, the number of overall requests can be expressed as follows:

$$
M=\sum_{i=1}^{n} M_{i}=\sum_{i=1}^{n}\left(M_{i z_{1}}+\ldots+M_{i z_{R}}\right)=\sum_{i=1}^{n} \sum_{r=1}^{R} M_{i z_{r}}
$$


In this case, based on $F_{i z_{r}}=M_{i z_{r}} / M_{i}, r=\overline{1, R}$, we can evaluate the social media activity of the patients by the medical fields according to the regions.

Based on $F_{i z_{r}}^{*}=\max \left\{F_{i z_{r}}, r=\overline{1, R}\right\}$, we can determine the region, which the patients most frequently refer from, by the medical fields (from which region and on which medical fields the patients send their requests).

10. Activity of e-patients by the medical fields according to their gender and region of residence. If we refer to the requests by the regions of Azerbaijan with regard to the gender of e-patients, then the overall requests can be summarized by the following formula:

$$
M=\sum_{i=1}^{n} M_{i}=\sum_{i=1}^{n}\left(M_{i z_{1}}+\ldots+M_{i z_{R}}\right)=\sum_{i=1}^{n} \sum_{r=1}^{R} M_{i z_{r}}=\sum_{i=1}^{n} \sum_{r=1}^{R}\left(M_{i q z_{r}}+M_{i k z_{r}}\right)
$$

In this case, based on $F_{i q z_{r}}=M_{i q z_{r}} / M_{i z_{r}}, r=\overline{1, R}$, we can determine the activity of female patients by $R$ regions, and based on $F_{i q z_{r}}^{*}=\max \left\{F_{i q z_{r}}, r=\overline{1, R}\right\}$, we can determine which medical field is most frequently referred by female patients (from which region and on which medical fields the female patients send their requests).

Based on $F_{i k z_{r}}=M_{i k z_{r}} / M_{i z_{r}}, r=\overline{1, R}$, we can determine the activity of female patients by $R$ regions, and based on $F_{i k z_{r}}^{*}=\max \left\{F_{i k z_{r}}, r=\overline{1, R}\right\}$, we can determine which medical field is most frequently referred by female patients (from which region and on which medical fields the female patients send their requests).

11. The social media activity by the fields of medicine with regard to the age and the region of residence of the female and male patients. If we refer to the requests to the medical fields with regard to the gender, age and region of residence of the patients, then the overall requests can be presented $\mathrm{s}$ as follows:

$$
\begin{aligned}
& M=\sum_{i=1}^{n} M_{i}=\sum_{i=1}^{n}\left(M_{i z_{1}}+\ldots+M_{i z_{R}}\right)=\sum_{i=1}^{n} \sum_{r=1}^{R} M_{i z_{r}}=\sum_{i=1}^{n} \sum_{r=1}^{R}\left(M_{i q z_{r}}+M_{i k z_{r}}\right)= \\
& =\sum_{i=1}^{n} \sum_{r=1}^{R}\left(\left(M_{i A q z_{r}}+M_{i A k z_{r}}\right)+\left(M_{i B q z_{r}}+M_{i B k z_{r}}\right)+\left(M_{i C q z_{r}}+M_{i C k z_{r}}\right)\right)
\end{aligned}
$$

In this case:

Based on $F_{i A q z_{r}}=M_{i A q z_{r}} / M_{i q z_{r}}, r=\overline{1, R}$, we can evaluate the social media activity of female patients from age group $A$ and the region $Z_{r}$ by the medical field $i$.

Based on $F_{i B q z_{r}}=M_{i B q z_{r}} / M_{i q z_{r}}, r=\overline{1, R}$, we can evaluate the social media activity of female patients from age group $B$ and the region $Z_{r}$ by the medical field $i$.

Based on $F_{i q C z_{r}}=M_{i C q z_{r}} / M_{i q z_{r}}, r=\overline{1, R}$, we can evaluate the social media activity of female patients from age group $C$ and the region $Z_{r}$ by the medical field $i$.

Based on $F_{i A k z_{r}}=M_{i A k z_{r}} / M_{i k z_{r}}, r=\overline{1, R}$, we can evaluate the social media activity of male patients from age group $A$ and the region $Z_{r}$ by the medical field $i$.

Based on $F_{i B k z_{r}}=M_{i B k z_{r}} / M_{i k z_{r}}, r=\overline{1, R}$, we can evaluate the social media activity of male patients from age group $B$ and the region $Z_{r}$ by the medical field $i$.

Based on $F_{i k C z_{r}}=M_{i k C z_{r}} / M_{i k z_{r}}, r=\overline{1, R}$, we can evaluate the social media activity of male patients from age group $C$ and the region $Z_{r}$ by the medical field $i$.

Based on $F_{i A q z_{r}}^{*}=\max \left\{F_{i A q z_{r}}, r=\overline{1, R}\right\}$, we can determine from which region female patients from age group $A$ most frequently refer to the medical field $i$.

Based on $F_{i B q z_{r}}^{*}=\max \left\{F_{i B q z_{r}}, r=\overline{1, R}\right\}$, we can determine from which region female patients from age group $B$ most frequently refer to the medical field $i$.

Based on $F_{i C q z_{r}}^{*}=\max \left\{F_{i C q z_{r}}, r=\overline{1, R}\right\}$, we can determine from which region female patients from age group $C$ most frequently refer to the medical field $i$.

Based on $F_{i A k z_{r}}^{*}=\max \left\{F_{i A k z_{r}}, r=\overline{1, R}\right\}$, we can determine from which region male patients from age group $A$ most frequently refer to the medical field $i$.

Based on $F_{i B k z_{r}}^{*}=\max \left\{F_{i B k z_{r}}, r=\overline{1, R}\right\}$, we can determine from which region male patients from age group $B$ most frequently refer to the medical field $i$. 
Based on $F_{i C k z_{r}}^{*}=\max \left\{F_{i C k z_{r}}, r=\overline{1, R}\right\}$, we can determine from which region male patients from age group $C$ most frequently refer to the medical field $i$.

\section{RESULTS AND DISCUSSION}

Formation of professional human resources in the professional medical social media space created a steady increase in the number of stakeholders where the forms of activities in this environment are diverse. From this point of view, new virtual relationships were formed. This information collected in a virtual space is a valuable source of information for improving the medical decision-making process in e-medical conditions. Referring to the results of the statistical analysis of the requests in this source, this article:

- defines the rating of physicians by the medical fields;

- defines the most frequently referred medical field in the country;

- defines the most frequently referred medical field by age;

- defines the most frequently referred medical field by gender;

- defines the most frequently referred medical fields by the regions of the country;

- defines the most frequently referred medical fields by the regions of the country, taking into account age and gender, and conceptually describes solution of similar problems.

Obviously, the approach proposed in the article also enables the improvement of decision-making process for the formation of public medicine, aimed at improving and protecting the health of the population, rather than individual patients. Further development of the research is aimed at determining the relations of the population's requests with the social, economic, environmental status, flora and fauna of the area where they live, and solving appropriate decision-making problems. This study aims to identify cause-and-effect relations between the population's health and the factors affecting it based on the territorial medical demographic indicators, and to solve decision-making problems.

An important aspect of the proposed approach is the use of the indicators of media activity of stakeholders and the informative indicators necessary for decision-making, based on the results of statistical analysis of the requests collected in the medical social media environment, rather than the content analysis. The process of medical decision-making based on these indicators is formally described for the first time.

The following issues are expected to be addressed through a comprehensive use of the indicators included in the following phases of the study:

1. Evaluation of medical service quality;

2. Comparison of digital divide of regions;

3. Selection of the best physician in different fields of medicine;

4. Identification of supply and demand for medical services in different areas of medicine;

5. Evaluation of medical security of the population by regions;

6. Identification of more favorable regions for living in terms of quality of medical services;

7. Medical social tomography of the regions;

8. Analysis of the medical infrastructure, ecological situation, population's lifestyle, socio-economic development in the regions, etc.

In the national medical social media space, it is expedient to address the following:

- expanding the medical content to involve many stakeholders in online medical resources;

- providing information on the territory of residence, age, gender, e-mail of the patients during registration in medical websites. The aim is to gather more information in the national medical social media environment to improve medical decision-making. Thus, current studies show that demographic indicators are important in assessing the health of the population (Cesare, Grant \& Hawkins, 2017). This approach enables the doctors to appoint the right diagnosis, and also shapes a valuable source of information for medical decisions based on different demographic indicators; 
- developing security measures to protect the confidentiality of the patient's data in medical media. Currently, in some medical networks, not only personal information of the patients, even the advice they got from doctors, their diagnosis, treatment method, etc. are accessible and handy to all. This poses a threat to the confidentiality of the personal and health information of e-patients. To overcome this problem, the patients can take advantage of the security policy (Jingquan, 2013).

\section{CONCLUSION}

The article proposed a conceptual approach to the use of the information gathered in the physicianpatient relations sector of social media in the medical decision-making process.

In this regard, the followings are solved:

- stakeholders and relations were analyzed to classify the data collected in the medical social media environment, and the social media environment was segmented for the different types of relations formed;

- the requests of e-patients in the physician-patient segment of social media was analyzed on the example of the online medicine consultation group of Facebook "Online medical consultation" and the medical community of Azerbaijan tibb.az, and, the results of statistical analysis were presented;

- Initial informative indicators for medical decision-making were developed based on the surveys implemented in the physician-patient segment of the social media environment of the country;

- referring to the introduced informative indicators and a number of demographic indicators of epatients, a conceptual mechanism for medical decision making was theoretically developed.

\section{REFERENCES}

Aksoy, M. E. (2018). A Qualitative Study on the Reasons for Social Media Addiction. European Journal of Educational Research, 7(4), 861-865. https://doi.org/10.12973/eu-jer.7.4.861

Alguliyev, R., Aliguliyev, R., \& Yusifov, F. (2018). Role of Social Networks in E-government: Risks and Security Threats. Online Journal of Communication and Media Technologies, 8(4), 363-376. https://doi.org/10.12973/ojcmt/3957

Amit, P., Tejashree, W., \& Swati R. M. (2014). Review of Online Product using Rule based and Fuzzy Logic. Smiley's International Journal of Computing and Technology, 1, 39-44.

Bhaskar S. (2017). Examining physican use of social media in 2017. P.M360 The essential resource for pharma marketers. Retrieved from www.pm360online.com/examining-physician-use-of-social-media-in-2017/

Bollen, J., Mao, H., \& Zeng X.-J. (2011). Twitter mood predicts the stock market. Journal of Computational Science, 2(1), 1-8. https://doi.org/10.1016/j.jocs.2010.12.007

Bridewell, W., \& Das, A. K. (2011). Social Network Analysis of Physician Interactions: The Effect of Institutional Boundaries on Breast Cancer Care. AMIA Annu Symp Proc. 152-160. https://www.ncbi.nlm.nih.gov/pmc/articles/PMC3243165/

Campanini, S. (2016). 24 Outstanding Statistics \& Figures on How Social Media has Impacted the Health Care Industry. Mashable, Linkedin, Retrieved from www.linkedin.com/pulse/24-outstanding-statistics-figureshow-social-media-has-campanini

Campbell, L., Evans, Y., Pumper, M., \& Moreno, M. A. (2016). Social media use by physicians: a qualitative study of the new frontier of medicine. BMC Medical Informatics and Decision Making, 16, 91. https://doi.org/10.1186/s12911-016-0327-y

Cesare, N., Grant, C. \& Hawkins, J. B. (2017). Demographics in Social Media Data for Public Health Research: Does it matter? Bloomberg Data for Good Exchange Conference. Retrieved from https://arxiv.org/ftp/arxiv/papers/1710/1710.11048.pdf

Chang, H., \& Choi, M. (2016). Big Data and Healthcare: Building an Augmented World. Healthcare informatics research, 22(3), 153-155. https://doi.org/10.4258/hir.2016.22.3.153

Dalal, M. K.\& Zaveri, M. A. (2014). Opinion Mining from online user reviews using Fuzzy Linguistic Hedges. Applied Computational Intelligence and So. Computing, 2014(735942), 1-9. https://doi.org/10.1155/2014/735942 
Efimenko, I. V., \& Horoshevskij, V. F. (2016). Online consultations in the medical field: knowledge extraction and analytics. Proceedings of the 15th National Conference on Artificial Intelligence with the international participation, 33-46. Retrieved from http://www.raai.org/resurs/papers/kii-2016/cai2016vol2.pdf

Fogelson, N. S., Rubin, Z. A., \& Ault, K. A. (2013). Beyond likes and tweets: an in-depth look at the physician social media landscape. Clinical Obstet Gynecol, 56(3), 495-508. https://doi.org/10.1097/GRF.0b013e31829e7638

Gallant, L. M., Irizarry, C., Boone, G., \& Kreps L. G. (2011). Promoting Participatory Medicine with Social Media: New Media Applications on Hospital Websites that Enhance Health Education and e-Patients' Voices. Journal of Participatory Medicine, 3. Retrieved from www.jopm.org/evidence/research/2011/10/31

Haque, Md., \& Rahman T. (2014). Sentiment analysis by using fuzzy logic. International Journal of Computer Science, Engineering and Information Technology (IJCSEIT), 4(1), 33-48. Retrieved from https://bmcmedinformdecismak.biomedcentral.com/articles/10.1186/s12911-016-0327-y

Islam, M. S., Hasan, M. M., Wang, X., Germack, H. D. \& Noor-E-Alam, M. (2018). A Systematic Review on Healthcare Analytics: Application and Theoretical Perspective of Data Mining. Healthcare (Basel), 6(2), 54. https://doi.org/10.3390/healthcare6020054

Jingquan, L. (2013). Privacy policies for health social networking sites. Journal of the American Medical Informatics Association, 4(20), 704-707. https://doi.org/10.1136/amiajnl-2012-001500

Keckley, P. H. (2010). Issue Brief: Social Networks in Health Care Communication, collaboration and insights. Produced by the Deloitte Center for Health Solutions. Retrieved from http://www.healthinformationand communicationsystems.pbworks.com/w/file/fetch/93972338/SM\%204b\%20Full.pdf

Khokhar, A. (2017). Social networking for healthcare professionals. Indian journal of medical Science, 69(1), 6366. https://doi.org/10.18203/issn.0019-5359.IndianJMedSci20170499

Krithika, R. D., \& Rosiline, J. B. (2017). Dynamic and Reliable Intelligent Data Mining Technique on Social Media Drug Related Posts. IEEE International Conference on Power, Control, Signals and Instrumentation Engineering (IEEE ICPCSI), 1788-1794.

Luneva, E. E., Efremov, A. A., \& Banokin, P. I. (2015). Automated assessment of emotions of users of social networks based on fuzzy logic. Economics, Statistics and Informatics, 3, $249-254$. https://doi.org/10.21686/2500-3925-2015-3-249-254

Mammadova, M. H., \& Isayeva, A. M. (2018). E-health activity in social media environment. Problems of information society, 1, 52-62. https://doi.org/10.25045/jpis.v09.i1.05

Mammadova, M. H., \& Jabrayilova, Z.G. (2019). Electronic medicine: formation and scientific-theoretical problems, Baku: "Information Technologies" publishing house, 319. Retrieved from https://ict.az/uploads/files/E-medicine-monograph-IIT-ANAS.pdf

Mammadova, M. H., Jabrayilova, Z. G., \& Isayeva, A. M. (2019). Analysis of physician-patient relations segment of social media: opportunities and challenges. Problems information society, 2, 41-50. https://doi.org/10.25045/jpis.v10.i2.04

Martino, I. D., D'Apolito, R., McLawhorn, A. S., Fehring, K. A., Sculco, P. K., \& Gasparini G. (2017). Social media for patients: benefits and drawbacks. Curr Rev Musculoskelet Med., 10(1), 141-14. https://doi.org/10.1007/s12178-017-9394-7

Mengxue, Z., Meizhuo, Z., \&, Chen, G. (2019). Automatic discovery of adverse reactions through Chinese social media. Data mining and knowledge discovery, 33(4), 848-870.

Nadali, S., Murad, M. A. A. \& Kadir, R. A. (2010). Sentiment classification of customer reviews based on fuzzy logic. Proceedings of the International Symposium on Information Technology (ITSim' 10), 1037-1044. https://doi.org/10.1109/ITSIM.2010.5561583

Simsek, A., Elciyar, K., \& Kizilhan, T. (2019). A Comparative Study on Social Media Addiction of High School and University Students. Contemporary Educational Technology, 10(2), 106-119. https://doi.org/10.30935/cet.554452

Swan, M. (2012). Crowdsourced Health Research Studies: An Important Emerging Complement to Clinical Trials in the Public Health Research Ecosystem. Journal of Medical Internet Research, 14(2), 46. https://doi.org/10.2196/jmir.1988

Tomar, D. \& Agarwal, S. (2013). A survey on Data Mining approaches for Healthcare. Int. J. Bio-Sci. Bio-Technol., 5, 241-266. https://doi.org/10.14257/ijbsbt.2013.5.5.25 
Tunc-Aksan, A., \& Akbay, S.E. (2019). Smartphone Addiction, Fear of Missing Out, and Perceived Competence as Predictors of Social Media Addiction of Adolescents. European Journal of Educational Research, 8(2), 559-569. https://doi.org/10.12973/eu-jer.8.2.559

Usak, M., Kubiatko, M., Shabbir, M. S., Viktorovna Dudnik, O., Jermsittiparsert, K., \& Rajabion, L. (2019). Health care service delivery based on the Internet of things: A systematic and comprehensive study. International Journal of Communication Systems, 33(2), e4179. https://doi.org/10.1002/dac.4179

Vo, A.-D., \& Ock, C.-Y. (2012). Sentiment classification: a combination of PMI, sentiWordNet and fuzzy function. Proceedings of the 4th International Conference on Computational Collective Intelligence Technologies and Applications (ICCCI '12), 7654 (2) of Lecture Notes in Computer Science, 373-382. https://doi.org/10.1007/978-3-642-34707-8_38

Zadeh, L. A. (1975). The concept of a linguistic variable and its application to approximate reasoning-II. Information Sciences, 8(4), 301-357. https://doi.org/10.1016/0020-0255(75)90046-8

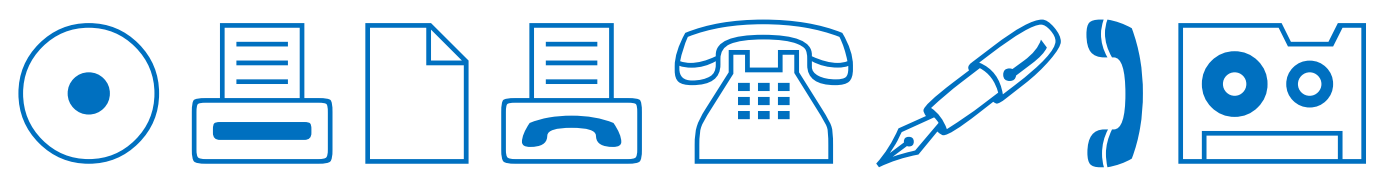

